da taxa de cicatrização. Devido às alterações da reparação tecidular e das funções imunológicas e vasculares, o sucesso da terapia endodôntica em pacientes com DM pode estar comprometido. O presente estudo tem como objetivo investigar se a presença de DM tipo 2 influencia os resultados do tratamento endodôntico. Materiais e métodos: Para este estudo retrospetivo, foram selecionados todos os tratamentos endodônticos realizados no Instituto de Endodontia, Faculdade de Medicina, entre 2015 e 2019. Após a aplicação dos critérios de inclusão (diabetes tipo 2 relatado na história clínica; ausência de outra doença sistémica; sem limite de idade e sem restrição do estado de saúde oral do paciente), todos os pacientes que respeitavam esses critérios foram incluídos no grupo diabético (GD). Para o grupo controlo (GC), foi selecionado aleatoriamente um número semelhante de pacientes sem nenhuma doença sistémica e que necessitaram de tratamento endodôntico na mesma instituição e no mesmo intervalo de tempo. No GC houve em consideração a variação de idade e sexo do GD para homogeneizar os grupos durante a randomização. Entre seis e quarenta meses após o tratamento endodôntico, uma consulta de controlo foi agendada para avaliar os parâmetros clínicos e radiográficos. O sucesso do tratamento endodôntico foi definido pela ausência de sinais clínicos e radiográficos na consulta de controlo, independentemente da presença ou ausência de lesão apical na radiografia pré-operatória. Os resultados foram avaliados pelo Modelo de Regressão de Cox e pelo Modelo de Kaplan-Meier. Resultados: Os resultados revelaram uma menor taxa de sucesso da terapia endodôntica em pacientes diabéticos tipo 2 com diferenças estatisticamente significativas entre os grupos (OR: 2.482; IC 95\%: 1.168-5.272; $\mathrm{p}=$ 0.018). Através do Modelo de Kaplan-Meier, observou-se que o GD necessitou de $30.35 \pm 2.78$ meses para atingir o sucesso endodôntico, enquanto o GC necessitou de $14.75 \pm 0.58$ meses $(\mathrm{p}<0.01)$. Conclusões: A presença de DM poderá diminuir a capacidade de reparação dos tecidos periapicais. De acordo com os resultados obtidos, e não relacionados à qualidade dos tratamentos realizados, é possível concluir que pacientes com DM tipo 2 podem ter menor taxa de sucesso no tratamento endodôntico do que indivíduos saudáveis.

http://doi.org/10.24873/j.rpemd.2020.12.795

\section{\#073 A hipossialia em pacientes polimedicados}

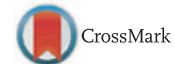

João Gato Marques*, Cecília Rozan, André Peixoto, Luís Proença, Ana Cristina Manso

Instituto Universitário Egas Moniz; CiiEM - Centro de Investigação Interdisciplinar Egas Moniz

Objetivos: Descrever a prevalência de hipossialia nos pacientes polimedicados, relacionando-a com variáveis sociodemográficas e dados clínicos da polimedicação. Materiais e métodos: Estudo transversal realizado numa amostra aleatória constituída por 40 indivíduos, de ambos os sexos e com idades entre 23 e 84 anos, que compareceram nas consultas de Medicina Dentária Preventiva e Comunitária entre fevereiro e março de 2020, na Clínica Dentária Egas Moniz. A recolha de dados foi feita através da aplicação de um questionário sobre variáveis sociodemográficas e questões de dados clínicos no âmbi- to da administração regular de, pelo menos, dois dos seguintes medicamentos: anticonvulsivantes, antidepressivos, antidiabéticos orais, anti-hipertensores e anti-histamínicos H1. Seguidamente efetuou-se a sialometria, para obtenção das taxas de fluxo salivar não estimulado e estimulado, considerando hipossialia quando a taxa de fluxo salivar não estimulado $<0,1$ $\mathrm{mL} / \mathrm{min}$ e/ou taxa de fluxo salivar estimulado $<0,7 \mathrm{~mL} / \mathrm{min}$. Os participantes assinaram o consentimento informado, garantindo-se a total confidencialidade dos dados. Os dados recolhidos neste estudo foram submetidos a uma análise estatística, pelos valores de prevalência, através do software IBM SPSS Statistics ${ }^{\circledR}$ V.24. Resultados: A prevalência da hipossialia da amostra foi $50 \%$, sendo mais prevalente no grupo $\geq 65$ anos $(27,5 \%)$, sexo feminino $(27,5 \%)$, caucasiana ( $45 \%)$, escolaridade até ao $1 .^{\circ}$ Ciclo (20\%), rendimento familiar mensal entre 1 e 2 salários mínimos nacionais (20\%), reformados(as) (32,5\%), casados(as)/união de facto (32,5\%) e não fumadores (45\%). Verificou-se a prevalência de hipossialia no grupo com administração concomitante de antidiabéticos orais e anti-hipertensores (20\%), com combinação de 2 medicamentos $(32,5 \%)$ e com um tempo de toma dos medicamentos $>10$ anos (20\%). Não foi encontrada associação da prevalência de hipossialia com variáveis sociodemográficas ( $p>0,05)$, nem com os dados clínicos da polimedicação $(p>0,05)$. Conclusões: Com este estudo foi possível observar que metade da amostra padece de hipossialia mas desconhece a sua repercussão na cavidade oral. Os resultados obtidos realçam a necessidade de se reforçar o diagnóstico precoce da hipossialia associada à polimedicação como um meio de promoção e prevenção de doenças orais futuras.

http://doi.org/10.24873/j.rpemd.2020.12.796

\#074 A Hipossialia em pacientes hipertensos

Patrícia Martins*, Cecília Rozan, Luís Proença, Cristina Manso

Instituto Universitário Egas Moniz; CiiEM - Centro de investigação interdisciplinar Egas Moniz

Objetivos: A Hipertensão Arterial é uma patologia sistémica prevalente em Portugal. Este estudo pretende descrever a prevalência de hipossialia na população que administra anti-hipertensores, relacionando-a com as variáveis sociodemográficas e com os dados clínicos da Hipertensão Arterial. Materiais e métodos: Estudo transversal com uma amostra aleatória constituída por 40 indivíduos, de ambos os sexos, de idade 41 a 90 anos que compareceram nas consultas de Medicina Dentária Preventiva e Comunitária entre fevereiro e março de 2020, na Clínica Universitária Egas Moniz. A recolha de dados foi feita através de um inquérito realizado por escrito com questões no âmbito dos dados clínicos da Hipertensão Arterial e das variáveis sociodemográficas. Seguidamente, mediu-se a tensão arterial e efetuou-se a sialometria, para obtenção das taxas de fluxo salivar não estimulado e estimulado. Esteve presente o consentimento informado e garantiu-se a total confidencialidade dos dados. Os dados recolhidos neste estudo foram submetidos a uma análise estatística descritiva pelos valores de prevalência através do software IBM SPSS Statistics ${ }^{\circledR}$ V.24. Resultados: A prevalência 
da hipossialia da amostra foi $60 \%$, sendo mais prevalente nos grupos de 51-60 anos (17,5\%) e 61-70 anos (17,5\%). A amostra é composta por $55 \%$ do sexo masculino e $45 \%$ do sexo feminino, sendo a hipossialia mais prevalente no sexo masculino (35\%), na raça caucasiana (55\%), que frequentou até o $1 .^{\circ}$ ciclo de escolaridade (20\%), que afere entre 1-2 salários mínimos nacionais (42,5\%), reformados (as) (40\%) e casados (as) / união de facto $(47,5 \%)$. Verificou-se a prevalência de hipossialia no grupo dos hipertensos $>10$ anos (37,5\%), que administram anti-hipertensores $>10$ anos $(37,5 \%)$, administram um único anti-hipertensor (52,5\%) e na administração dos Bloqueadores dos Canais de Cálcio (22,5\%). Não foi encontrada correlação entre prevalência de hipossialia e variáveis sociodemográficas ( $p>0,05)$, nem com os dados clínicos da Hipertensão ( $p>0,05)$. Conclusões: Com este estudo foi possível observar que mais que metade da nossa amostra padece de hipossialia, mas desconhece a sua repercussão na cavidade oral. Os resultados obtidos realçam a necessidade de se reforçar o diagnóstico precoce da hipossialia associada a administração de anti-hipertensores como um meio de promoção e prevenção de doenças orais futuras.

http://doi.org/10.24873/j.rpemd.2020.12.797

\section{\#075 Caracterização da Escovagem dos Dentes na População Pré-Escolar}

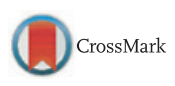

\section{Diana Ferreira*, Mário Bernardo, Sónia Mendes}

Faculdade de Medicina Dentária da Universidade de Lisboa

Objetivos: O objetivo deste estudo foi caracterizar a escovagem dos dentes da população em idade pré-escolar, residente em Portugal. Materiais e métodos: A população-alvo foi constituída por encarregados de educação de crianças em idade pré-escolar, que frequentavam jardins de infância portugueses. A recolha de dados foi realizada através de um questionário online. O questionário, construído para o efeito com base na revisão da literatura, permitiu a obtenção de informação sociodemográfica, da escovagem em ambiente familiar e da escovagem em ambiente escolar. Foi realizada a análise descritiva dos dados e utilizados os testes do Qui-quadrado, Exato de Fisher, Mann-Whitney e Kruskal-Wallis $\alpha=0,05)$. Resultados: A amostra foi constituída por 711 participantes. A maioria das crianças realizava a escovagem dos dentes em casa $(99,4 \%)$, duas ou mais vezes por dia $(65,3 \%)$ ou com ajuda de um adulto (62,9\%). Grande parte das crianças não realizava escovagem dos dentes no jardim de infância (71,2\%). As principais barreiras identificadas relativamente à autorização da escovagem no jardim de infância foram a falta de higiene e segurança $(32,6 \%)$ e a possibilidade de partilha de escovas $(42,9 \%)$. Considerando a escovagem dos dentes nos dois contextos, familiar e escolar, a percentagem de escovagem bidiária foi referida em três quartos das crianças. No entanto, apenas $7,8 \%$ realizavam corretamente todos os procedimentos associados à escovagem. A escovagem dos dentes em ambiente escolar foi referida como sendo mais frequente nos jardins de infância privados $(p<0,001)$ e na região Autónoma dos Açores $(p<0,001)$. Um maior nível de instrução do encarregado de educação contribuiu significativamente para a não autoriza- ção da escovagem no jardim de infância $(p=0,019)$ e para a realização dos procedimentos corretos de escovagem $(p=0,007)$. Também a visita ao profissional de saúde oral se verificou associada à realização dos procedimentos corretos de escovagem dos dentes $(\mathrm{p}=0,005)$. Conclusões: A escovagem bidiária dos dentes revelou-se bastante frequente na população estudada, no entanto a frequência de crianças que realiza todos os procedimentos corretos de escovagem dos dentes verificou-se baixa, bem como a frequência de escovagem dos dentes em ambiente escolar.

http://doi.org/10.24873/j.rpemd.2020.12.798

\section{\#076 Avaliação da Utilização da Fotografia em Medicina Dentária Durante a Pandemia da Covid-19}

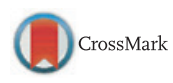

Bruno Seabra*, Teresa Albuquerque, Henrique Luís, Jaime Portugal

Faculdade de Medicina Dentária da Universidade de Lisboa

Objetivos: Analisar a adaptação e mudança de comportamentos na utilização da fotografia em Medicina Dentária na atual situação pandémica de COVID-19. Materiais e métodos: Foi criado um inquérito no Google forms e que foi partilhado para médicos dentistas por mensagem ou por e-mail, entre os dias 13-08-2020 e 15-09-2020. Foi usada uma técnica de amostragem não-probabilística. Responderam ao inquérito 235 médicos dentistas que realizam a sua prática clínica em Portugal. Foram analisadas variáveis demográficas e comportamentais. Os dados foram recolhidos e exportados para análise no programa SPSS - Statistical Package for Social Sciences (versão 27.0). Resultados: Os resultados mostram que entre os 235 médicos dentistas que preencheram o inquérito, $80,4 \%$ já usavam a fotografia na sua prática clínica antes da pandemia e apenas 19,6\% responderam que não utilizavam. Até dia 15 de Setembro, dos que costumam fotografar, cerca 98,9\% já tinha reiniciado o seu trabalho clínico. No entanto, cerca de 15\% não se encontrava ainda a trabalhar no seu horário normal. Cerca de $40 \%$ referiram que a prática clínica teria sido afetada pela diminuição do número de horas de consultas e correspondentemente pelo número de pacientes por dia. Apenas $21 \%$ consideraram que teria havido uma diminuição acentuada no número de pacientes a procurar consulta. Apenas $19 \%$ dos médicos dentistas que fotografam os seus casos por rotina, consideraram que a COVID-19 tivesse afetado de forma importante a fotografia no seu dia a dia. Notou-se uma diminuição no número de casos fotografados, principalmente para quem fotografa menor número de casos. Apenas 8,6 $\%$ referem ter deixado de fotografar os seus casos. Diferentes causas foram apontadas mas a mais importante para $46 \%$ foi a tentativa em diminuir risco de infeção cruzada. Para evitar contaminação, 53\% referem que a medida mais importante que realizam é a desinfeção da camara após sua utilização. Apenas $12 \%$ assumem ter tomado como medida face ao COVID-19, isolar a câmara sempre que a utilizam ou ter sempre uma pessoa responsável para fotografar os casos. Conclusões: A pandemia da COVID-19 trouxe algumas alterações ao tempo de consulta e disponibilidade de agenda para atender 